\title{
Toll-Like Receptor 3 in Liver Diseases
}

\author{
Shi Yin ${ }^{1,2}$ and Bin Gao ${ }^{1}$ \\ ${ }^{1}$ Laboratory of Liver Diseases, National Institute on Alcohol Abuse and Alcoholism, National Institutes of Health, Bethesda, \\ 5625 Fishers Lane, MD 20892, USA \\ ${ }^{2}$ Department of Geriatrics, The Affiliated Provincial Hospital of Anhui Medical University, Hefei, Anhui 230001, China
}

Correspondence should be addressed to Bin Gao, bgao@mail.nih.gov

Received 27 April 2010; Accepted 14 July 2010

Academic Editor: Ekihiro Seki

Copyright ( $) 2010$ S. Yin and B. Gao. This is an open access article distributed under the Creative Commons Attribution License, which permits unrestricted use, distribution, and reproduction in any medium, provided the original work is properly cited.

Toll-like receptor 3 (TLR3) is a member of the TLR family that can recognize double-stranded RNA (dsRNA), playing an important role in antiviral immunity. Recent studies have shown that TLR3 is also expressed on parenchymal and nonparenchymal cells in the liver as well as on several types of immune cells. In this review, we summarize the role of TLR3 in liver injury, inflammation, regeneration, and liver fibrosis, and discuss the implication of TLR3 in the pathogenesis of human liver diseases including viral hepatitis and autoimmune liver disease.

\section{Introduction}

TLR3 is a member of the Toll-like receptor family that can recognize double-stranded RNA (dsRNA) from viruses, endogenous dsRNA from dying cells, or synthetic dsRNA polyriboinosinic:polyribocytidylic acid (poly I:C). TLR3 expression has been found in endosomal compartments (such as dendritic cells and macrophages) or at the cell surfaces (such as human fibroblasts). Binding of TLR3 and its ligand leads to conformational changes in the TLR3 cytoplasmic tail, followed by recruitment of TIR domaincontaining adaptor inducing IFN- $\beta$ (TRIF), and subsequent activation of the mitogen activated protein (MAP) kinase pathway, the NF- $\kappa \mathrm{B}$ family of transcription factors, and the IFN regulatory factor (IRF) family of transcription factors, which then induce interferon (IFN) and inflammatory cytokine production $[1,2]$. It is generally believed that TLR3 plays an important role in host response to viruses via recognizing dsRNA; however, its role in antiviral immunity has been questioned by some in vivo studies $[3,4]$. The controversial reports on the role of TLR3 in the antiviral defense may be due to the difference in the type of viruses, the type of cells that are infected, the viral load, its model of infection (endoplasmic versus cytoplasmic), and stage of infection. Recent studies have shown that TLR3 also plays important roles in the pathophysiology of a variety of liver diseases [5-7], which may attribute to the wide expression of TLR3 on all types of liver cells, including hepatocytes [8-10], stellate cells [11], sinusoidal endothelial cells [12], Kupffer cells, biliary epithelial cells $[13,14]$, as well as immune cells such as NK cells, NKT cells [15], and liver lymphocytes [12]. In this review, we summarize the recent findings regarding the role of TLR3 in liver injury, inflammation, regeneration, fibrosis, viral infection, and autoimmune liver disease.

\section{TLR3 in Liver Inflammation and Injury}

It has been noticed for many years that injection of mice with the TLR3 ligand poly I:C induced significantly liver inflammation with a predominant accumulation of $\mathrm{NK}$ cells $[16,17]$. Recent studies suggest that such NK cell accumulation is due to the recruitment of NK cells from the spleen, which is regulated positively by the expression of chemokines and the presence of T cells [18], but regulated negatively by the $\beta 2$ integrin CD11b [19]. Treatment with poly I:C induced mild liver injury in normal mice via an NK cell-dependent manner [20] but induced massive liver necrosis in D-galactosamine (D-GalN)-sensitized mice [21, $22]$. In the model of liver injury induced by poly I:C/DGalN, it is believed that injection of these two reagents elevates the expression of retinoic acid early inducible-1 (Rae-1) on Kupffer cells. Upregulated Rae-1 acts as an NK 
cell stimulating ligand to activate NK cells via targeting NKG2D receptor on NK cells. Activated NK cells then produce a large amount of IFN- $\gamma$, which acts in synergy with Kupffer cell-derived TNF- $\alpha$ to induce massive hepatocellular damage [22]. In addition, poly I:C treatment also induced significantly liver injury in transgenic mice with HBV surface antigen (HBs-B6) $[23,24]$. It was shown that depletion of NK cells or blockage of IFN- $\gamma$ but not depletion of Kupffer cells or neutralization of IL-12 diminished the poly I:C-induced liver injury in HBs-B6 mice, suggesting that NK cells/IFN- $\gamma$ contribute to the pathogenesis of liver injury in this model. In contrast to the detrimental effect of poly I:C on liver injury, pretreatment with poly I:C had a beneficial effect to reduce the mortality and liver injury induced by lipopolysaccharide plus D-GalN in mice [25]. This protective effect of poly I:C seems to be mediated via poly I:C downregulation of TLR4 expression on Kupffer cells/macrophages and subsequent reduction of the responsiveness of Kupffer cells/macrophages to LPS stimulation. In addition, activation of TLR3 on $\mathrm{V} \alpha 14$ iNKT cells may also negatively regulate liver inflammation via preventing intrahepatic $\gamma \delta \mathrm{T}$ cell accumulation [15].

Although the effect of poly I:C on liver injury has been extensively investigated, the role of TLR3 signaling in these effects remains obscure. TLR3-deficient mice had reduced response to poly I:C stimulation, reduced production of inflammatory cytokines stimulated by poly I:C, and resistance to poly I:C/D-GalN-induced mortality, suggesting a critical role of TLR3 signaling in poly I:C-mediated liver injury [21]. The important role of TLR3 in liver inflammation and injury has also been recently revealed in Concanavalin A- (Con A-) induced T cell hepatitis model by using TLR3-deficient mice [12]. Injection of Con A markedly increased TLR3 expression on liver lymphocytes and sinusoidal endothelial cells. Disruption of the TLR3 gene abolished Con A-induced liver injury. Finally, by using chimeric mice, Xiao et al. [12] demonstrated that TLR3 signaling in both nonhematopoietic and hematopoietic cells plays a critical role in the pathogenesis of Con A-induced $\mathrm{T}$ cell hepatitis. However, what the endogenous ligands are and how these ligands activate TLR3 in this Con A-induced T cell hepatitis model remain unknown.

\section{TLR3 in Liver Regeneration}

Liver regeneration is a very complicated process orchestrated with a series of signaling cascades induced by cytokines, growth factors and hormones [26]. Current knowledge from experimental studies of liver regeneration suggests that tissue loss or cell damage triggers innate immune responses and initiates liver regeneration. Among the multiple innate components, the role of TLR4 signaling in liver regeneration has been extensively investigated. However, the results on the role of TLR4 signaling and its adapter protein myeloid differentiation factor-88 (MyD88) in liver regeneration have been controversial [27, 28]. Early studies showed that TLR4/MyD88 pathway was critical for the initiation of liver regeneration [27]. Impaired liver regeneration was observed in $\mathrm{MyD} 88^{-/-}$mice after partial hepatectomy (PHx). This phenomenon was associated with grossly subnormal induction of the expression of immediate early genes involved in hepatocyte replication and the phosphorylation of STAT3 in the liver, and the reduced production of TNF- $\alpha /$ IL- 6 by the activation of NF- $\kappa \mathrm{B}$ in the Kupffer cells. Surprisingly, a later report indicated liver regeneration was not suppressed in mice deficient in MyD88, TLR2, TLR4, or CD14 gene [28]. Although normal hepatocyte DNA replication was observed in Myd88 knockout mice, PHx-mediated induction of proinflammatory cytokines TNF- $\alpha$, IL- 6 , and their downstream signaling pathways was reduced in MyD-88 knockout mice [28]. At present, the reasons for the discrepancy between these 2 studies are not clear $[27,28]$. The different surgical techniques and animal facility environment may contribute to the different findings on liver regeneration in MyD88 knockout mice.

In contrast, a series of evidence show that TLR3/IFN$\gamma /$ STAT1 axis plays an inhibitory role in liver regeneration, suggesting that the innate immune system may play an important role in balancing liver regeneration [29-32]. We have previously demonstrated that after infection with murine cytomegalovirus (MCMV) or poly(I:C) injection, NK cells are activated and produce IFN- $\gamma$ that in turn attenuates liver regeneration after PHx. Depletion of NK cells or disruption of either the IFN- $\gamma$ gene or the IFN$\gamma$ receptor gene enhances liver regeneration and partially abolishes the negative effects of MCMV and poly(I:C) on liver regeneration. These results suggest that viral infection and the TLR3 ligand negatively regulate liver regeneration via activation of innate immunity (NK/IFN- $\gamma$ ) [31]. Consistent with these results of the inhibitory role of TLR3 in liver regeneration, TLR3 ${ }^{-/-}$mice demonstrated earlier hepatocyte proliferation and an increase in liver regeneration following PHx [29]. In the absence of TLR3, hepatocyte proliferation was accelerated while the levels of IL-6 and soluble interleukin-6 receptor (sIL-6R) were reduced. TLR3 signaling was induced in hepatocytes at the early time points after $\mathrm{PHx}$, resulting in enhanced $\mathrm{NF}-\kappa \mathrm{B}$ activation, the increase levels of Rip3 and activation of caspase-8, with no evidence of apoptosis. These findings suggest TLR3 signaling plays an important role in inhibiting liver regeneration [29].

We have further demonstrated that IFN- $\gamma$ inhibits liver regeneration via activation of STAT1 and subsequent induction of IRF-1 and p21 [30, 32]. Disruption of the STAT1 gene abolished poly I:C suppression of liver regeneration and the inhibitory effect of poly $\mathrm{I}: \mathrm{C}$ on liver regeneration was diminished in IRF- $1^{-/-}$and $\mathrm{p} 21 \mathrm{cip} 1^{-/-}$mice. Treatment with IFN- $\gamma$ in vitro inhibited cell proliferation of wildtype mouse hepatocytes, but not STAT $1^{-1-}$ hepatocytes. The inhibitory effect of IFN- $\gamma$ on cell proliferation was also diminished in $\mathrm{IRF}-1^{-/-}$and $\mathrm{p} 21 \mathrm{cip} 1^{-/-}$hepatocytes, but enhanced in SOCS1 ${ }^{-/-}$hepatocytes. Hepatocyte proliferation was unaffected by treatment with poly I:C alone, but when hepatocytes were cocultured with liver lymphocytes, hepatocyte proliferation was inhibited by IFN- $\gamma /$ STAT1dependent mechanisms. Moreover, in HCV-infected livers with cirrhosis, activation of STAT1 was detected and correlated positively with liver injury but correlated negatively with hepatocyte proliferation. 


\section{TLR3 in Liver Fibrosis}

Liver fibrosis is a common response to virtually all forms of chronic liver injury and is characterized with hepatic stellate cell (HSC) activation and accumulation of extracellular matrix proteins $[33,34]$. HSCs are generally believed to be the most important cells in producing collagens and contributing to the pathogenesis of liver fibrosis. Activation of HSCs is controlled by many cytokines and growth factors. Among them, TGF- $\beta$ is considered the most important factor to induce HSC transformation while PDGF plays a critical role in stimulating HSC proliferation. Recent studies have also suggested that TLR4 plays an important role in promoting liver fibrosis via enhancing TGF$\beta$ signaling in HSCs [35]. Our laboratory has demonstrated that treatment of mice with the TLR3 ligand poly I:C markedly inhibits liver fibrosis [36, 37], which was confirmed later by another laboratory [38]. The studies from our laboratory as well as other laboratories suggest that multiple mechanisms contribute to poly I:C-mediated inhibition of liver fibrosis. First, poly I:C treatment induces NK cell activation, and activated NK cells then kill earlyactivated or senescence-activated HSCs that have increased expression of NK cell activating ligands [36, 38]; Second, poly I:C treatment induces NK cells to produce IFN- $\gamma$ that subsequently induces HSC apoptosis and cell cycle arrest [37]. Recent studies from Dr. Schlaak's laboratory have shown that poly I:C treatment stimulates HSCs that express high levels of TLR3 to produce type I IFN- $\beta$ [11]. Since type I IFN is known to inhibit HSC proliferation [39], it is plausible to speculate that activation of TLR3 may also directly block HSC proliferation via production of IFN- $\beta$, thereby contributing to suppression of liver fibrosis. Furthermore, we have demonstrated that chronic alcohol consumption suppresses poly I:C-mediated activation of NK cell activation and induction of cytotoxic mediators on liver lymphocytes [40] and poly I:C inhibition of liver fibrosis [41]. Alcohol inhibition of poly I:C-mediated activation of NK cells is probably mediated via suppression of poly I:C activation of TLR3 signaling on NK cells $[42,43]$. Finally, abrogation of the antifibrotic effect of NK cells by alcohol may be an important mechanism contributing to alcohol acceleration of liver fibrosis in patients with viral infection [41]. We have previously reported that injection of poly I:C inhibits liver regeneration induced by partial hepatectomy [31]. Interestingly, a recent paper shows that injection of poly I:C also inhibits liver regeneration induced by administration of single dose of CCl4 [44]. However, it is not clear whether poly I:C also has inhibitory effect on liver regeneration in a model of liver fibrosis induced by chronic $\mathrm{CCl} 4$ treatment, and whether inhibition of liver regeneration by TLR3 also contributes to liver fibrogenesis.

\section{TLR3 in Viral Hepatitis Infection}

TLR3 is generally believed to play an important role in the innate immune response against viral infection, including viral hepatitis infection, although controversial results have been reported [4]. The antiviral effects of TLR3 signaling on viral hepatitis infection are likely mediated via stimulating of a variety of cells to produce type I IFN that subsequently inhibits HCV or HBV replication [11, 45-47]. These cells include HSCs [11], monocyte-derived dendritic cells [48], hepatocytes [47], Kupffer cells [46], sinusoidal endothelial cells [46], NK cells [49], and so forth. Recent studies showed that TLR3 can also directly sense HCV infection in human hepatocytes, acting independently of retinoic acid-inducible gene I (RIG-I), followed by activation of IRF-3 and ISGs that suppress HCV replication [10]. However, several lines of evidence suggest that TLR3 signaling is suppressed during viral hepatitis infection, which may contribute to the escape of hepatitis virus from the surveillance of innate immunity and lead to the chronic infection. First, expression of TLR3 and type I IFN was significantly decreased in monocytederived dendritic cells from patients with chronic HBV or acute-on-chronic HBV liver failure compared with normal healthy individuals. Such reduction correlated positively with severity of the disease [50]; Second, infection of hepatoma cells with HCV in vitro degraded TRIF protein, an essential TLR3 adaptor, and subsequently attenuated poly I:C-induced signaling [10]; Third, the NS3/4A serine protease of hepatitis $\mathrm{C}$ virus (HCV) can interrupt TLR3 and/or RIG-I-mediated signal transduction by proteolytic cleavage of TRIF and/or CADIF [51-53]. The TLR3 pathway plays an important role in influencing host innate immunity and viral clearance during viral hepatitis infection, and may represent a useful therapeutic approach for the treatment of viral hepatitis. However, a greater understanding of the specific cellular source of TLR3 signals and TLR3 pathway changes in different stages of viral infection may assist in the design of appropriate therapeutic interventions that target this TLR3 pathway in patients with chronic viral hepatitis infection.

The association between TLR3 gene polymorphisms and chronic $\mathrm{HCV}$ infection was also recently investigated [54]. Two single nucleotide polymorphisms (SNPs) were identified within the TLR3 gene: rs5743305 (T/A) is located within the promoter region; rs3775291 (C/T) is located within exon 4. Both SNPs were not found to be associated with TLR3 gene expression in peripheral blood mononuclear cells (PBMCs); however, a tendency of higher TLR3 gene expression in the liver was found for exon 4 TT genotypes. No association was found between both SNPs and the clinical parameters of disease progression of chronic HCV infection, but the TLR3 exon genotype was found to be related to resistance to HCV subtype Ia. These studies suggest that the TLR3 SNP associated with higher TLR3 expression in the liver might be related to the resistance to $\mathrm{HCV}$ subtype Ia infection but has no role in disease progression after a chronic infection is established.

\section{TLR3 in Autoimmune Liver Disease}

TLR3, together with other endosomal TLRs (TLR7 and TLR9), have been implicated in the pathogenesis of a variety of autoimmune diseases [1], including primary 


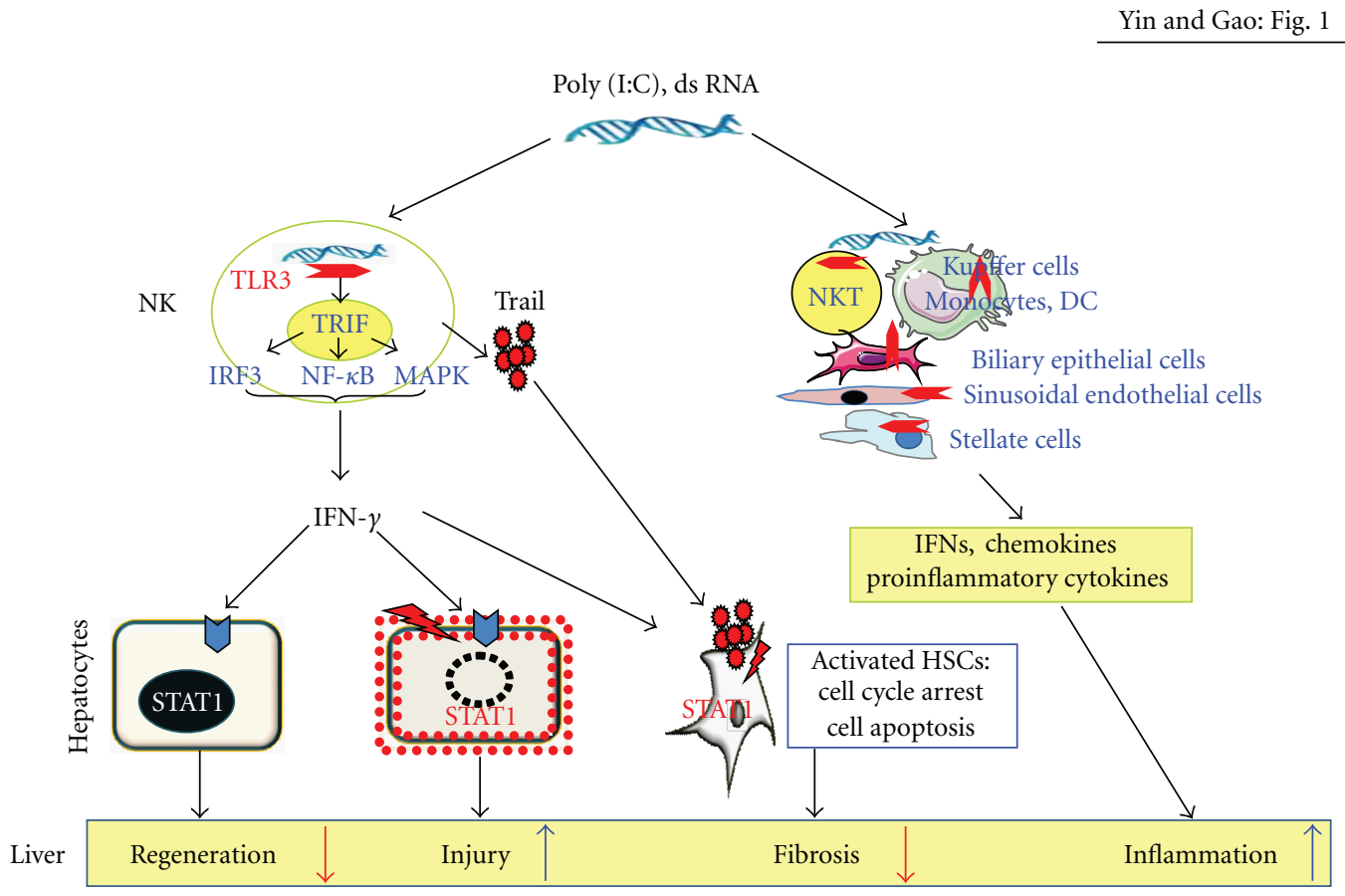

FIgure 1: A model depicting the roles of TLR3 in liver injury, inflammation, regeneration, and fibrosis. Poly I:C or dsRNA binds TLR3 receptor in NK cells and stimulates NK cells to produce IFN- $\gamma$ that induces hepatocyte cell cycle arrest and apoptosis via activation of STAT1. IFN- $\gamma$ also induces stellate cell cycle arrest and apoptosis in an STAT1-depdent manner, resulting in inhibition of liver fibrosis. Poly I:C can also inhibit liver fibrosis via activation of NK cell killing of early activated stellate cells. In addition, poly I:C or dsRNA targets TLR3 in many other cell types, followed by production of IFNs, chemokines, and anti-inflammatory cytokines, resulting in liver inflammation.

biliary cirrhosis. The critical role of TLR3 in autoimmune hepatitis was clearly demonstrated in a model of hepatitis induced by infection of lymphocytic choriomeningitis virus (LCMV) [55]. This study suggests that poly I:C activation of TLR3 on antigen-presentation cells such as macrophages and dendritic cells produce type I IFN and TNF- $\alpha$, which then trigger the release of CXCL9 by hepatocytes, Kupffer cells, endothelial cells, and so forth. CXCL9 then attracts CXCR3 positive self-reactive $\mathrm{CD} 8+\mathrm{T}$ cells to kill hepatocytes, resulting in autoimmune hepatitis. However, the role of TLR3 in the pathogenesis of human autoimmune diseases is less clear. Immunohistochemistry analyses showed that the expression of TLR3 was markedly increased in biliary epithelial cells at sites of ductular reaction in primary biliary cirrhosis and autoimmune hepatitis $[13,14]$. A strong positive correlation between the mRNA levels of TLR3 and type I IFN in the liver was found in the patients with primary biliary cirrhosis, suggesting TLR3 signaling is involved in the pathogenesis of primary biliary cirrhosis [14]. In animal models, injection of poly I:C induced primary biliary cirrhosis-like cholangitis (such as infiltration of mononuclear cells and elevation of AMA autoantibodies) in a genetically susceptible mouse strain of female C57BL/6 mice $[56,57]$. At present, the mechanisms by which poly I:C treatment induces cholangitis remain obscure. It is believed that poly I:C activation of TLR3 signaling in biliary epithelial cells as well as hepatocytes and immune cells in the liver results in production of type
I IFN, which subsequently contributes to the pathogenesis of primary biliary cirrhosis $[13,14,58]$. However, a recent study suggests that poly I:C induction of type I IFN in biliary epithelial cells is mediated via targeting RIG-I and melanoma differentiation-associated gene 5 (MDA5), not TLR3 [13]. Further studies are required to clarify the role of TLR3 in primary biliary cirrhosis.

In summary, TLR3 is expressed on parenchymal and nonparenchymal cells in the liver as well as in many types of immune cells including macrophages, dendritic cells, NK cells, NKT cells, and so forth (see Figure 1). In general, activation of TLR3 by dsRNA induces NK cell accumulation and activation in the liver, leading to liver inflammation and injury. TLR3 signaling also negatively regulates liver regeneration via stimulating NK cells to produce IFN- $\gamma$ that subsequently induces hepatocyte death and cell cycle arrest via an STAT1-dependent manner. Activation of NK cells by the TLR3 ligand poly I:C inhibits liver fibrosis via killing of activated stellate cells and producing IFN- $y$ that subsequently induces stellate cell apoptosis and inhibits stellate cell proliferation. Clinical studies suggest that TLR3 may contribute to the resistance to HCV subtype Ia infection but seems to have no role in disease progression after a chronic infection is established. TLR3 has also been implicated in the autoimmune liver disease in animal models, but more studies are required to clarify the role of TLR3 in human autoimmune diseases. 


\section{Abbreviations}

D-GalN: D-galactosamine

HSC: Hepatic stellate cells

IFN: Interferon

MAPK: Mitogen activated protein kinase

MyD-88: Myeloid differentiation factor-88

Poly I:C: Polyriboinosinic:polyribocytidylic acid (poly I:C)

TLR3: Toll-like receptor 3

TRIF: $\quad$ TIR domain-containing adaptor inducing IFN- $\beta$ (TRIF).

\section{Acknowledgments}

This work was supported by the intramural program of NIAAA, NIH. No conflicts of interest exist for all authors.

\section{References}

[1] S. Trivedi and E. L. Greidlinger, "Endosomal Toll-like receptors in autoimmunity: mechanisms for clinical diversity," Therapy, vol. 6, no. 3, pp. 433-442, 2009.

[2] E. F. Kenny and L. A. J. O'Neill, "Signalling adaptors used by Toll-like receptors: an update," Cytokine, vol. 43, no. 3, pp. 342-349, 2008.

[3] E. Vercammen, J. Staal, and R. Beyaert, "Sensing of viral infection and activation of innate immunity by toll-like receptor 3," Clinical Microbiology Reviews, vol. 21, no. 1, pp. 13-25, 2008.

[4] M. Schröder and A. G. Bowie, "TLR3 in antiviral immunity: key player or bystander?" Trends in Immunology, vol. 26, no. 9, pp. 462-468, 2005.

[5] E. Seki and D. A. Brenner, "Toll-like receptors and adaptor molecules in liver disease: update," Hepatology, vol. 48, no. 1, pp. 322-335, 2008.

[6] G. Szabo, A. Dolganiuc, and P. Mandrekar, "Pattern recognition receptors: a contemporary view on liver diseases," Hepatology, vol. 44, no. 2, pp. 287-298, 2006.

[7] A. Mencin, J. Kluwe, and R. F. Schwabe, "Toll-like receptors as targets in chronic liver diseases," Gut, vol. 58, no. 5, pp. 704720, 2009.

[8] E. Khvalevsky, L. Rivkin, J. Rachmilewitz, E. Galun, and H. Giladi, "TLR3 signaling in a hepatoma cell line is skewed towards apoptosis," Journal of Cellular Biochemistry, vol. 100, no. 5, pp. 1301-1312, 2007.

[9] K. Li, Z. Chen, N. Kato, M. Gale Jr., and S. M. Lemon, "Distinct poly(I-C) and virus-activated signaling pathways leading to interferon- $\beta$ production in hepatocytes," Journal of Biological Chemistry, vol. 280, no. 17, pp. 16739-16747, 2005.

[10] N. Wang, Y. Liang, S. Devaraj, J. Wang, S. M. Lemon, and K. $\mathrm{Li}$, "Toll-like receptor 3 mediates establishment of an antiviral state against hepatitis C virus in hepatoma cells," Journal of Virology, vol. 83, no. 19, pp. 9824-9834, 2009.

[11] B. Wang, M. Trippler, R. Pei et al., "Toll-like receptor activated human and murine hepatic stellate cells are potent regulators of hepatitis C virus replication," Journal of Hepatology, vol. 51, no. 6, pp. 1037-1045, 2009.

[12] X. Xiao, P. Zhao, D. Rodriguez-Pinto et al., "Inflammatory regulation by TLR3 in acute hepatitis," Journal of Immunology, vol. 183, no. 6, pp. 3712-3719, 2009.
[13] M. Nakamura, K. Funami, A. Komori et al., "Increased expression of Toll-like receptor 3 in intrahepatic biliary epithelial cells at sites of ductular reaction in diseased livers," Hepatology International, vol. 2, no. 2, pp. 222-230, 2008.

[14] Y. Takii, M. Nakamura, M. Ito et al., "Enhanced expression of type I interferon and toll-like receptor-3 in primary biliary cirrhosis," Laboratory Investigation, vol. 85, no. 7, pp. 908-920, 2005.

[15] T. Gardner, Q. Chen, Y. Jin, and M. Ajuber, "Toll-like receptor 3 ligand dampens liver inflammation by stimulating Valpha14 invariant natural killer T cells to negatively regulate gamma/delta T cells," American Journal of Pathology, vol. 176, no. 5, pp. 1779-1789, 2010.

[16] R. H. Wiltrout, B. J. Mathieson, and J. E. Talmadge, "Augmentation of organ-associated natural killer activity by biological response modifiers. Isolation and characterization of large granular lymphocytes from the liver," Journal of Experimental Medicine, vol. 160, no. 5, pp. 1431-1449, 1984.

[17] R. H. Wiltrout, A. M. Pilaro, M. E. Gruys et al., "Augmentation of mouse liver-associated natural killer activity by biologic response modifiers occurs largely via rapid recruitment of large granular lymphocytes from the bone marrow," Journal of Immunology, vol. 143, no. 1, pp. 372-378, 1989.

[18] J. Wang, J. Xu, W. Zhang, H. Wei, and Z. Tian, "TLR3 ligandinduced accumulation of activated splenic natural killer cells into liver," Cellular Molecular of Immunology, vol. 2, no. 6, pp. 449-453, 2005.

[19] M. Zhang, Y. Han, C. Han et al., “The beta2 integrin CD11b attenuates polyinosinic:polycytidylic acid-induced hepatitis by negatively regulating natural killer cell functions," Hepatology, vol. 50, no. 5, pp. 1606-1616, 2009.

[20] Z. Dong, H. Wei, R. Sun, Z. Hu, B. Gao, and Z. Tian, "Involvement of natural killer cells in PolyI:C-induced liver injury," Journal of Hepatology, vol. 41, no. 6, pp. 966-973, 2004.

[21] L. Alexopoulou, A. C. Holt, R. Medzhitov, and R. A. Flavell, "Recognition of double-stranded RNA and activation of NFB by Toll-like receptor 3," Nature, vol. 413, no. 6857, pp. 732$738,2001$.

[22] X. Hou, R. Zhou, H. Wei, R. Sun, and Z. Tian, "NKG2Dretinoic acid early inducible- 1 recognition between natural killer cells and Kupffer cells in a novel murine natural killer cell-dependent fulminant hepatitis," Hepatology, vol. 49, no. 3, pp. 940-949, 2009.

[23] Y. Chen, R. Sun, W. Jiang, H. Wei, and Z. Tian, "Liverspecific HBsAg transgenic mice are over-sensitive to Poly(I:C)induced liver injury in NK cell- and IFN- $\gamma$-dependent manner," Journal of Hepatology, vol. 47, no. 2, pp. 183-190, 2007.

[24] Y. Chen, H. Wei, R. Sun, Z. Dong, J. Zhang, and Z. Tian, "Increased susceptibility to liver injury in hepatitis B virus transgenic mice involves NKG2D-ligand interaction and natural killer cells," Hepatology, vol. 46, no. 3, pp. 706-715, 2007.

[25] W. Jiang, R. Sun, H. Wei, and Z. Tian, “Toll-like receptor 3 ligand attenuates LPS-induced liver injury by down-regulation of toll-like receptor 4 expression on macrophages," Proceedings of the National Academy of Sciences of the United States of America, vol. 102, no. 47, pp. 17077-17082, 2005.

[26] G. K. Michalopoulos, "Liver regeneration," Journal of Cellular Physiology, vol. 213, no. 2, pp. 286-300, 2007.

[27] E. Seki, H. Tsutsui, Y. Iimuro et al., "Contribution of Toll-like receptor/myeloid differentiation factor 88 signaling to murine liver regeneration," Hepatology, vol. 41, no. 3, pp. 443-450, 2005. 
[28] J. S. Campbell, K. J. Riehle, J. T. Brooling, R. L. Bauer, C. Mitchell, and N. Fausto, "Proinflammatory cytokine production in liver regeneration is Myd88-dependent, but independent of Cd14, Tlr2, and Tlr4," Journal of Immunology, vol. 176, no. 4, pp. 2522-2528, 2006.

[29] E. Zorde-Khvalevsky, R. Abramovitch, H. Barash et al., "Toll-like receptor 3 signaling attenuates liver regeneration," Hepatology, vol. 50, no. 1, pp. 198-206, 2009.

[30] K. Shen, S.-S. Zheng, O. Park, H. Wang, Z. Sun, and B. Gao, "Activation of innate immunity (NK/IFN- $\gamma$ ) in rat allogeneic liver transplantation: contribution to liver injury and suppression of hepatocyte proliferation," American Journal of Physiology, vol. 294, no. 4, pp. G1070-G1077, 2008.

[31] R. Sun and B. Gao, "Negative regulation of liver regeneration by innate immunity (natural killer cells/interferon- $\gamma$ )," Gastroenterology, vol. 127, no. 5, pp. 1525-1539, 2004.

[32] R. Sun, O. Park, N. Horiguchi et al., "STAT1 contributes to dsRNA inhibition of liver regeneration after partial hepatectomy in mice," Hepatology, vol. 44, no. 4, pp. 955-966, 2006.

[33] R. Bataller and D. A. Brenner, "Liver fibrosis," Journal of Clinical Investigation, vol. 115, no. 4, pp. 209-218, 2005.

[34] S. L. Friedman, "Mechanisms of Hepatic Fibrogenesis," Gastroenterology, vol. 134, no. 6, pp. 1655-1669, 2008.

[35] E. Seki, S. De Minicis, C. H. Österreicher et al., "TLR4 enhances TGF- $\beta$ signaling and hepatic fibrosis," Nature Medicine, vol. 13, no. 11, pp. 1324-1332, 2007.

[36] S. Radaeva, R. Sun, B. Jaruga, V. T. Nguyen, Z. Tian, and B. Gao, "Natural killer cells ameliorate liver fibrosis by killing activated stellate cells in NKG2D-dependent and tumor necrosis factor-related apoptosis-inducing ligand-dependent manners," Gastroenterology, vol. 130, no. 2, pp. 435-452, 2006.

[37] W.-I. Jeong, O. Park, S. Radaeva, and B. Gao, "STAT1 inhibits liver fibrosis in mice by inhibiting stellate cell proliferation and stimulating NK cell cytotoxicity," Hepatology, vol. 44, no. 6, pp. 1441-1451, 2006.

[38] V. Krizhanovsky, M. Yon, R. A. Dickins et al., "Senescence of activated stellate cells limits liver fibrosis," Cell, vol. 134, no. 4, pp. 657-667, 2008.

[39] A. Mallat, A.-M. Preaux, S. Blazejewski, J. Rosenbaum, D. Dhumeaux, and P. Mavier, "Interferon alfa and gamma inhibit proliferation and collagen synthesis of human Ito cells in culture," Hepatology, vol. 21, no. 4, pp. 1003-1010, 1995.

[40] H.-N. Pan, R. Sun, B. Jaruga, F. Hong, W.-H. Kim, and B. Gao, "Chronic ethanol consumption inhibits hepatic natural killer cell activity and accelerates murine cytomegalovirus-induced hepatitis," Alcoholism: Clinical and Experimental Research, vol. 30, no. 9, pp. 1615-1623, 2006.

[41] W. Jeong, O. Park, and B. Gao, "Abrogation of the antifibrotic effects of natural killer cells/interferon- $\gamma$ contributes to acohol acceleration of liver fibrosis," Gastroenterology, vol. 134, no. 1, pp. 248-258, 2008.

[42] P. Hébert and S. B. Pruett, "Ethanol suppresses polyinosinic:polycytidylic acid-induced activation of natural killer cells primarily by acting on natural killer cells, not through effects on other cell types," Alcohol, vol. 28, no. 2, pp. 75-81, 2002.

[43] S. D. Collier and S. B. Pruett, "Mechanisms of suppression of poly I:C-induced activation of NK cells by ethanol," Alcohol, vol. 21 , no. 1, pp. 87-95, 2000.

[44] H. Wei, H. Wei, H. Wang, Z. Tian, and R. Sun, "Activation of natural killer cells inhibits liver regeneration in toxin-induced liver injury model in mice via a tumor necrosis factor-alphadependent mechanism," American Journal of Physiology, vol. 299, no. 1, pp. G275-G282, 2010.
[45] M. Isogawa, M. D. Robek, Y. Furuichi, and F. V. Chisari, "Tolllike receptor signaling inhibits hepatitis B virus replication in vivo," Journal of Virology, vol. 79, no. 11, pp. 7269-7272, 2005.

[46] J. Wu, M. Lu, Z. Meng et al., "Toll-like receptor-mediated control of HBV replication by nonparenchymal liver cells in mice," Hepatology, vol. 46, no. 6, pp. 1769-1778, 2007.

[47] T. Kanda, R. Steele, R. Ray, and R. B. Ray, "Hepatitis C virus infection induces the beta interferon signaling pathway in immortalized human hepatocytes," Journal of Virology, vol. 81, no. 22, pp. 12375-12381, 2007.

[48] L. Perrin-Cocon, S. Agaugué, O. Diaz et al., "Th 1 disabled function in response to TLR4 stimulation of monocytederived DC from patients chronically-infected by Hepatitis C virus," PLoS One, vol. 3, no. 5, Article ID e2260, 2008.

[49] D. Duluc, F. Tan, M. Scotet et al., "PolyI:C plus IL-2 or IL12 induce IFN- $\gamma$ production by human NK cells via autocrine IFN- $\beta$," European Journal of Immunology, vol. 39, no. 10, pp. 2877-2884, 2009.

[50] N. Li, Q. Li, Z. Qian, Y. Zhang, M. Chen, and G. Shi, "Impaired TLR3/IFN- $\beta$ signaling in monocyte-derived dendritic cells from patients with acute-on-chronic hepatitis B liver failure: relevance to the severity of liver damage," Biochemical and Biophysical Research Communications, vol. 390, no. 3, pp. 630635, 2009.

[51] K. Li, E. Foy, J. C. Ferreon et al., "Immune evasion by hepatitis $\mathrm{C}$ virus NS3/4A protease-mediated cleavage of the Toll-like receptor 3 adaptor protein TRIF," Proceedings of the National Academy of Sciences of the United States of America, vol. 102, no. 8, pp. 2992-2997, 2005.

[52] E. Meylan, J. Curran, K. Hofmann et al., "Cardif is an adaptor protein in the RIG-I antiviral pathway and is targeted by hepatitis C virus," Nature, vol. 437, no. 7062, pp. 1167-1172, 2005.

[53] E. Foy, K. Li, R. Sumpter Jr. et al., "Control of antiviral defenses through hepatitis $\mathrm{C}$ virus disruption of retinoic acid-inducible gene-I signaling," Proceedings of the National Academy of Sciences of the United States of America, vol. 102, no. 8, pp. 2986-2991, 2005.

[54] E. Askar, R. Bregadze, J. Mertens et al., “TLR3 gene polymorphisms and liver disease manifestations in chronic hepatitis C," Journal of Medical Virology, vol. 81, no. 7, pp. 1204-1211, 2009.

[55] K. S. Lang, P. Georgiev, M. Recher et al., "Immunoprivileged status of the liver is controlled by Toll-like receptor 3 signaling," Journal of Clinical Investigation, vol. 116, no. 9, pp. 2456-2463, 2006.

[56] C. Okada, F. Akbar, N. Horiike, and M. Onji, "Early development of primary biliary cirrhosis in female C57BL/6 mice because of poly I:C administration," Liver International, vol. 25, no. 3, pp. 595-603, 2005.

[57] T. Jiang, Z. Han, S. Chen et al., "Resistance to activationinduced cell death and elevated FLIPL expression of CD4+ $\mathrm{T}$ cells in a polyI:C-induced primary biliary cirrhosis mouse model," Clinical and Experimental Medicine, vol. 9, no. 4, pp. 269-276, 2009.

[58] T. K. Mao, Z.-X. Lian, C. Selmi et al., "Altered monocyte responses to defined TLR ligands in patients with primary biliary cirrhosis," Hepatology, vol. 42, no. 4, pp. 802-808, 2005. 


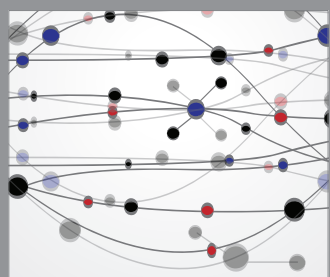

The Scientific World Journal
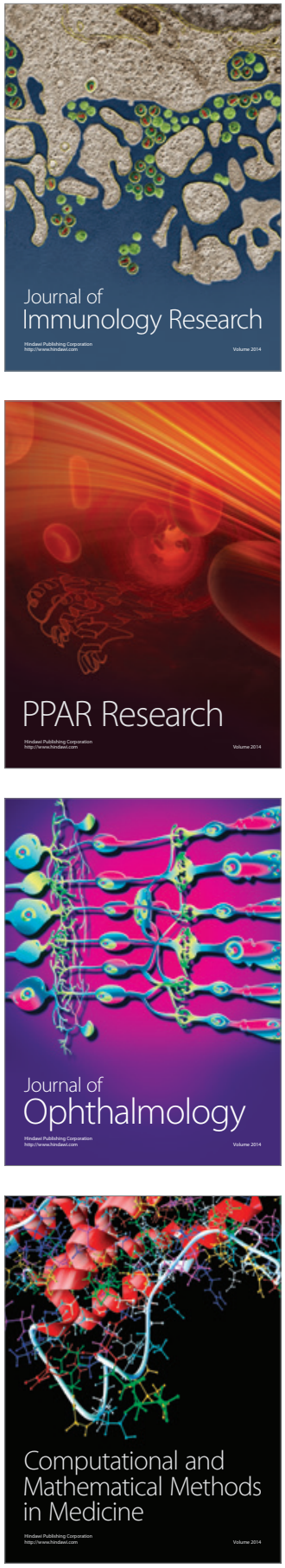

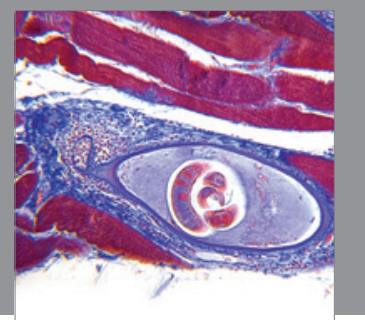

Gastroenterology

Research and Practice
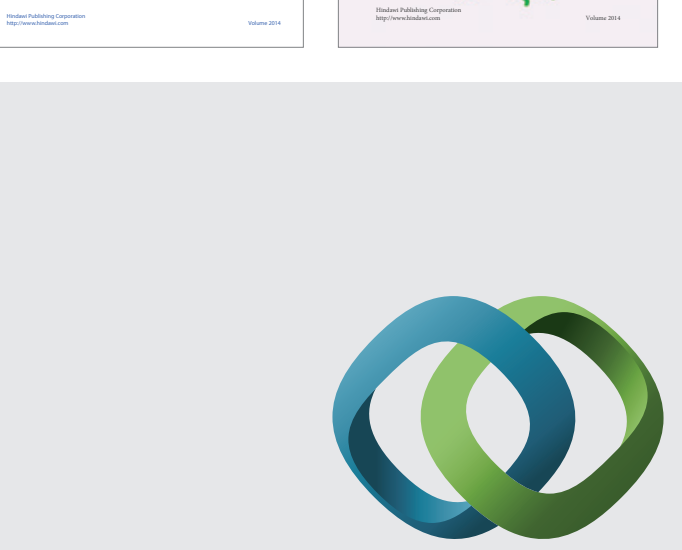

\section{Hindawi}

Submit your manuscripts at

http://www.hindawi.com
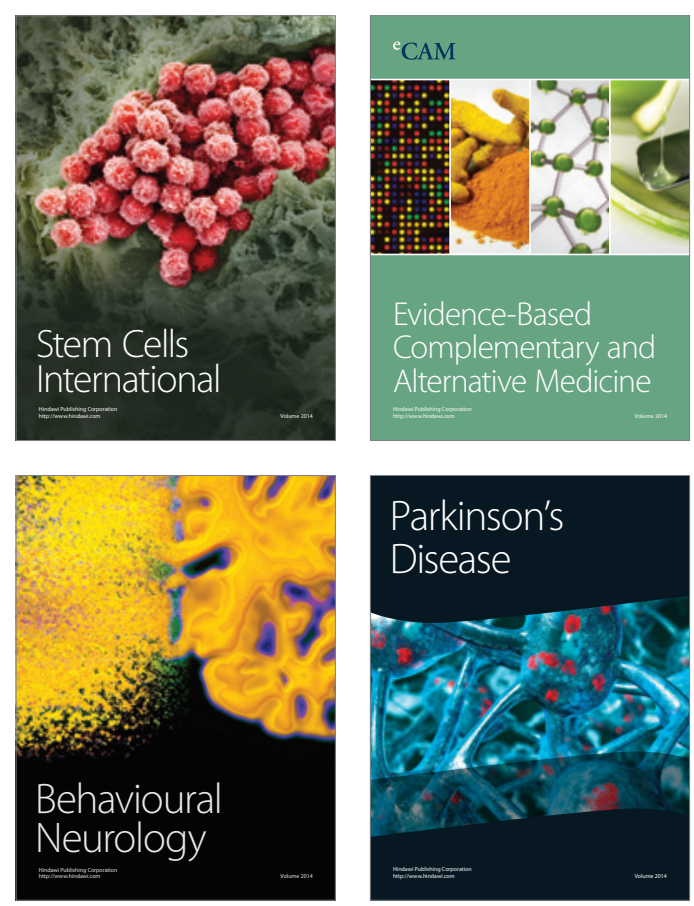

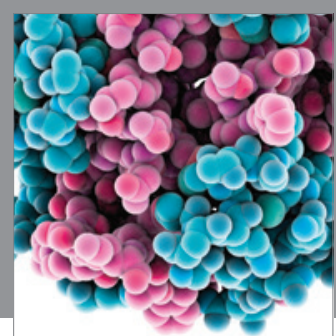

Journal of
Diabetes Research

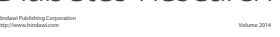

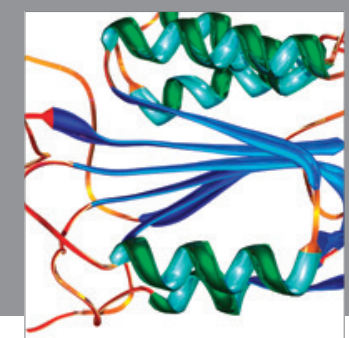

Disease Markers
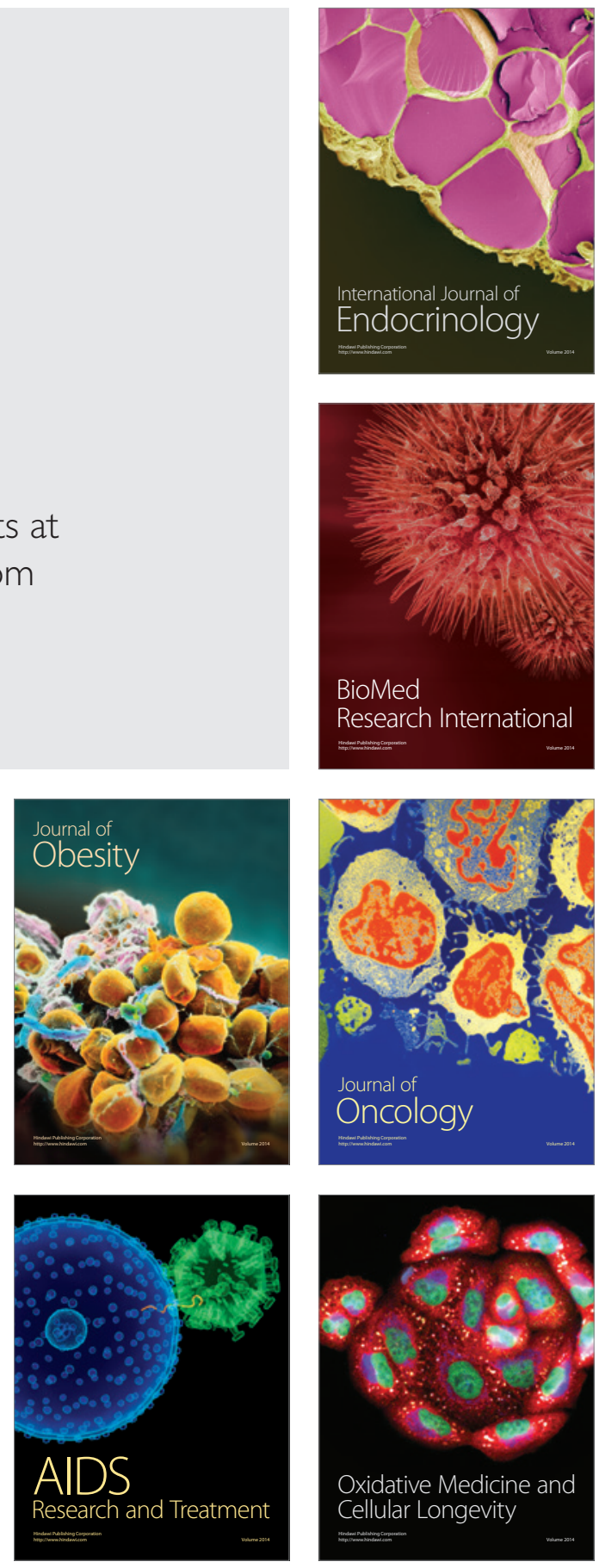